\title{
Frequency of Myeloid-derived Suppressor Cells in the Peripheral Blood Reflects the Status of Tumor Recurrence
}

\author{
TOMOKO TANAKA ${ }^{1}$, MITSUGU FUJITA ${ }^{2}$, HIROSHI HASEGAWA ${ }^{1}$, AKIRA ARIMOTO ${ }^{1}$, \\ MASAYASU NISHI ${ }^{1}$, EIJI FUKUOKA ${ }^{1}$, YUTAKA SUGITA ${ }^{1}$, TAKERU MATSUDA ${ }^{1}$, \\ YASUO SUMI ${ }^{1}$, SATOSHI SUZUKI ${ }^{1}$, YOSHIHIRO KAKEJI ${ }^{1}$ and KIMIHIRO YAMASHITA ${ }^{1}$ \\ ${ }^{1}$ Department of Surgery, Division of Gastrointestinal Surgery, \\ Kobe University Graduate School of Medicine, Kobe, Japan; \\ ${ }^{2}$ Department of Microbiology, Kindai University Faculty of Medicine, Osaka, Japan
}

\begin{abstract}
Background: Malignant tumors inhibit antitumor immune responses, which are driven by T-regulatory cells or myeloid-derived suppressor cells (MDSCs). Since MDSCs are involved in invasion, migration, and metastasis of tumor cells, we hypothesized that MDSCs are also involved in tumor recurrence after surgical resection. Materials and Methods: C57BL/6 mice were subcutaneously inoculated with B16F10 melanoma cells in the right flank. In some experiments, established tumors were surgically resected. Peripheral blood was drawn over time, and immune cells and cytokines were evaluated using flow cytometry. Results: MDSCs and relevant pro-inflammatory cytokines increased in the peripheral blood of tumor-bearing mice. Moreover, the frequency of MDSCs rapidly increased in mice with tumor recurrence. Conclusion: The frequency of MDSCs in the peripheral blood of tumor-bearing mice reflects the status of tumor progression as well as tumor recurrence. Continuous monitoring of MDSCs in the peripheral blood might be a useful indicator of tumor recurrence.
\end{abstract}

Despite recent development of antitumor modalities based on surgery, chemotherapy, and radiotherapy, it remains difficult to completely control tumor progression. This is, at least partially, attributable to tumor-induced immunosuppression;

Correspondence: Kimihiro Yamashita, MD, Ph.D., Department of Surgery, Division of Gastrointestinal Surgery, Kobe University Graduate School of Medicine, 7-5-2 Kusunoki-cho, Chuo-ku, Kobe, Hyogo 650-0017, Japan. Tel: +81 783825925, Fax: +81 783825939, e-mail: kiyama@med.kobe-u.ac.jp and Mitsugu Fujita, MD, Ph.D., Department of Microbiology, Kindai University Faculty of Medicine, 377-2 Ohno-Higashi, Osaka-Sayama, Osaka 589-8511, Japan. Tel: +81 723660221, Fax: +81 723660206, e-mail: mfujita47@gmail.com

Key Words: MDSC, tumor recurrence, pro-inflammatory cytokine, antitumor immunity. malignant tumors proactively inhibit antitumor immune responses in order to limit therapeutic efficacy and therefore maintain their survival and achieve progression (1). Immune cells responsible for this phenomenon include T-regulatory cells (Tregs) (2) and myeloid-derived suppressor cells (MDSCs) $(3,4)$, both of which possess strong proangiogenic and immunosuppressive capacities. To solve this issue, therapeutic strategies targeting immune checkpoints such as cytotoxic T-lymphocyte antigen 4 (CTLA4), programmed death 1 (PD1), or programmed death ligand 1 (PDL1) have been introduced into this field and led to favorable outcomes (5). Nevertheless, malignant tumors eventually escape from the effects of these therapies (6). Therefore, it is of a great importance to develop further strategies to cancel tumorinduced immunosuppression.

As mentioned above, MDSCs suppress antitumor immunity and promote tumor progression $(3,4)$. They also stimulate angiogenesis and promote vascular remodeling. Physiologically, MDSCs are heterogeneous immature myeloid cells that are unable to differentiate into mature macrophages, dendritic cells, and granulocytes. Murine MDSCs are positive for CD11b and granulocyte differentiation antigen (Gr1) and classified into two distinct subsets: CD11b ${ }^{\text {hi }} \mathrm{Gr} 1^{\text {mid }}{ }^{\text {Ly }} 6 \mathrm{C}^{\text {hi }}$ Ly $6 \mathrm{G}^{\text {lo }}$ monocytic MDSCs (mMDSCs) and CD11 $\mathrm{b}^{\mathrm{hi}} \mathrm{Gr} 1^{\mathrm{mid}} \mathrm{Ly} 6 \mathrm{C}^{\mathrm{lo}} \mathrm{Ly} 6 \mathrm{G}^{\mathrm{hi}}$ granulocytic MDSCs (gMDSCs). In humans with malignant tumors, gMDSCs express $\mathrm{CD}_{1} 5^{+} \mathrm{CD} 14^{-} \mathrm{CD} 11 \mathrm{~b}^{+} \mathrm{CD} 33^{+} \mathrm{HLA}-\mathrm{DR}^{-}$ whereas mMDSCs express $\mathrm{CD} 15^{-} \mathrm{CD} 14^{+} \mathrm{CD} 11 \mathrm{~b}^{+} \mathrm{CD} 33^{+}$ HLA-DR ${ }^{-}$. Morphologically, gMDSCs resemble neutrophils, whereas mMDSCs have typical monocytic features. With the presence of tumors, gMDSCs increase systemically whereas the proportion of mMDSCs stays consistent.

In order to achieve complete cure of malignant tumors, it is of the upmost importance to control tumor recurrence. To this end, there is a great demand for indicators to determine or predict tumor recurrence promptly; such indicators would allow individual tailor-made therapeutic strategies. Currently, 
several novel methods have been proposed (7). In addition to these, given that MDSCs are known to be involved in invasion, migration, and metastasis of tumor cells (4), we hypothesized that MDSCs are also involved in tumor recurrence after surgical resection.

\section{Materials and Methods}

Mice and cell lines. The study procedure was described previously (8). Briefly, pathogen-free female C57BL/6 mice (5-8 weeks old) were purchased from CLEA Japan (Tokyo, Japan). All mice were maintained under pathogen-free conditions and used in accordance with the institutional guidelines of Kobe University (approval number: P170308). The B16F10 murine melanoma cell line was purchased from American Type Culture Collection (Manassas, VA, USA) and maintained in RPMI-1640 medium supplemented with $10 \%(\mathrm{v} / \mathrm{v})$ heat-inactivated fetal bovine serum, $2 \mathrm{mM}$ glutamine, $100 \mathrm{U} / \mathrm{ml}$ penicillin, and $100 \mu \mathrm{g} / \mathrm{ml}$ streptomycin (Sigma-Aldrich, St Lois, $\mathrm{MO}, \mathrm{USA}$ ) at $37^{\circ} \mathrm{C}$ in $5 \% \mathrm{CO}_{2}$. Cultured cells were tested and found to be negative for mycoplasma and viral contamination.

Subcutaneous tumor inoculation and tumor size measurement. The procedure was described previously (9). Briefly, $1 \times 10^{7} \mathrm{~B} 16 \mathrm{~F} 10$ cells per mouse were s.c. injected into the right flank of C57BL/6 mice. Major and minor axes of s.c. tumors were measured by caliper every 3 days, and the tumor volume was calculated by multiplication of these values.

Surgical tumor removal. The procedure described previously (9) was applied with minor modifications. Briefly, s.c. tumors were surgically resected in some experiments as follows. The tumorbearing mice received isoflurane for anesthesia and ketoprofen (6 $\mathrm{mg} / \mathrm{kg}$ body weight) for analgesia on day 12 after tumor cell inoculation. Once the mice became immobilized, the skin above the s.c. tumors were shaved and sterilized with isopropanol. The tumors were completely resected en bloc with substantial surgical margins. Wounds were closed with silk suture. The mice were transferred to a warm, dry area and monitored until recovery.

Immune cell preparation. The procedure was described previously (9). Briefly, red blood cells (RBCs) were removed from the peripheral blood using RBC lysis buffer $(155 \mathrm{mM} \mathrm{NH} 4 \mathrm{Cl}$ in $10 \mathrm{mM}$ Tris-HCl buffer, $\mathrm{pH} 7.5,25^{\circ} \mathrm{C}$ ). The cells were passed through cell strainers to obtain single-cell suspension. The single-cell suspension was centrifuged and resuspended in $200 \mu \mathrm{l}$ PBS containing $1 \%$ bovine serum albmin (BSA) for $15 \mathrm{~min}$ at $4^{\circ} \mathrm{C}$. The cells were then stained with the antibodies described below.

Antibodies and flow cytometry. The procedure was as described previously $(10,11)$. The following anti-mouse monoclonal antibodies were purchased from BD Biosciences (San Jose, CA, USA): antiCD11b, anti-Gr1, anti-Ly6C, anti-CD11b, anti-Ly6G, anti-CD3, antiCD4, anti-CD25, anti-CD11c, and anti-B220. Anti-mouse CD45 and anti-mouse CD8 were purchased from BioLegend (San Diego, CA, USA). Cells were stained in a 96-round-bottom-well plate for 20-30 min at $4^{\circ} \mathrm{C}$ and washed with PBS containing $1 \%$ BSA. Flow cytometric data were obtained using a FACSVerse (Becton Dickinson, San Jose, CA, USA) and analyzed using FlowJo software (TreeStar, Ashland, OR, USA). All the cells were gated with CD45.
Cytometric bead array (CBA) assay. The procedure was as described previously (12). Briefly, plasma was collected from the peripheral blood of the tumor-bearing mice and aliquoted into polypropylene microcentrifuge tubes and stored at $-80^{\circ} \mathrm{C}$ until use. Concentrations of different cytokines were quantitatively measured using beads that were coated with antibodies against the following mouse cytokines: granulocyte colony-stimulating factor (GCSF), monocyte chemoattractant protein 1 (MCP1), tumor necrosis factor alpha (TNF $\alpha$ ), interleukin 6 (IL6), Interferon $\gamma$ (IFN $\gamma$ ), and interleukin 17A (IL17A). Stained samples were run on a FACSVerse (Becton Dickinson) instrument. All the samples were assayed in duplicate and quantitated using a standardized curve. The data were analyzed using FACSArray (BD Biosciences, San Jose, CA, USA).

Statistical analysis. The procedure has been described previously $(9,11)$. Briefly, one-way analysis of variance with Holm's post-hoc test was performed to analyze differences among multiple groups using JMP software (SAS Institute Inc, Cary, NC, USA). A value of $p<0.05$ was considered statistically significant.

\section{Results}

MDSCs substantially increased in the peripheral blood of tumor-bearing mice. To address the impact of tumor presence on the systemic cellular immunity, we extensively evaluated immune cells subsets in the peripheral blood by flow cytometry (Figure 1). Tumor-bearing mice exhibited a significant increase in the frequency of $\mathrm{CD}_{1} 1 \mathrm{~b}^{+} \mathrm{Gr} 1^{+}$MDSCs in their peripheral blood with tumor progression over time $(p<0.001$ for day $0 v s$. day 19; Figure 1A). In contrast to the MDSCs, other immune cell subsets such as $\mathrm{CD}^{+} \mathrm{T}$-cells, $\mathrm{CD}^{+}{ }^{+}$T-cells, Tregs, B-cells, and dendritic cells exhibited no association with the tumor progression over time (Figure 1BD). These data suggest that the frequency of MDSCs in the peripheral blood preferentially reflects the status of tumor progression in vivo compared with other immune cell subsets.

Granulocytic MDSCs preferentially increased in tumorbearing mice. MDSCs are known to have at least two distinct subsets: gMDSCs and mMDSCs (4). We, therefore, sought to evaluate the MDSC subsets in detail (Figure 2). On day 12 after tumor cell inoculation, the frequency of gMDSCs in total MDSCs exhibited a rapid increase compared with that of mMDSCs $(p<0.001)$. Thereafter, the frequency of gMDSCs gradually increased whereas mMDSCs remained almost at the initial level ( $p<0.001$ for day $0 v s$. day 19). These data suggest that the changes in the $\mathrm{CD}_{11} \mathrm{~b}^{+} \mathrm{Gr} 1^{+} \mathrm{MDSC}$ frequency primarily reflect the changes in gMDSCs.

MDSC-relevant pro-inflammatory cytokines increased in tumor-bearing mice. The findings that MDSCs would reflect the status of tumor progression in vivo led us to evaluate plasma levels of pro-inflammatory and inflammatory cytokines that are responsible for MDSC kinetics (3). To this end, we performed CBA-based cytokine monitoring. The 
A
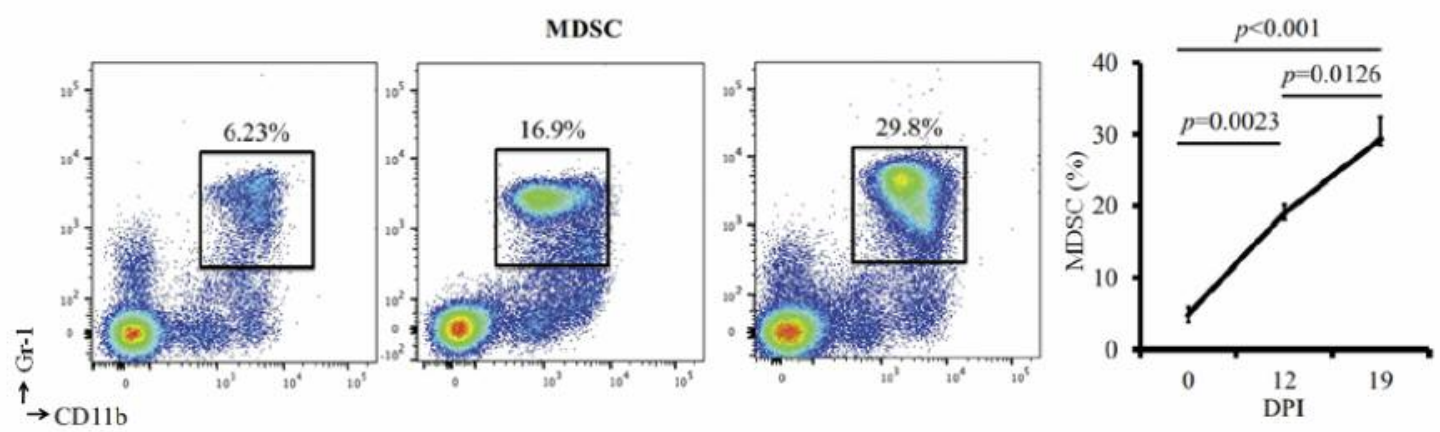

B
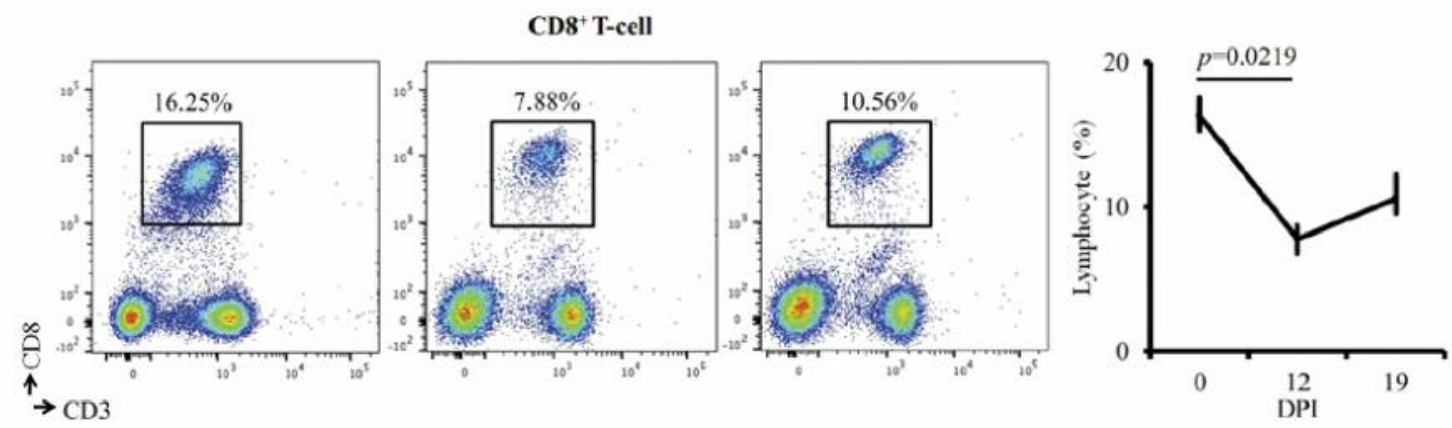

C

CD4 $4^{+}$-cell
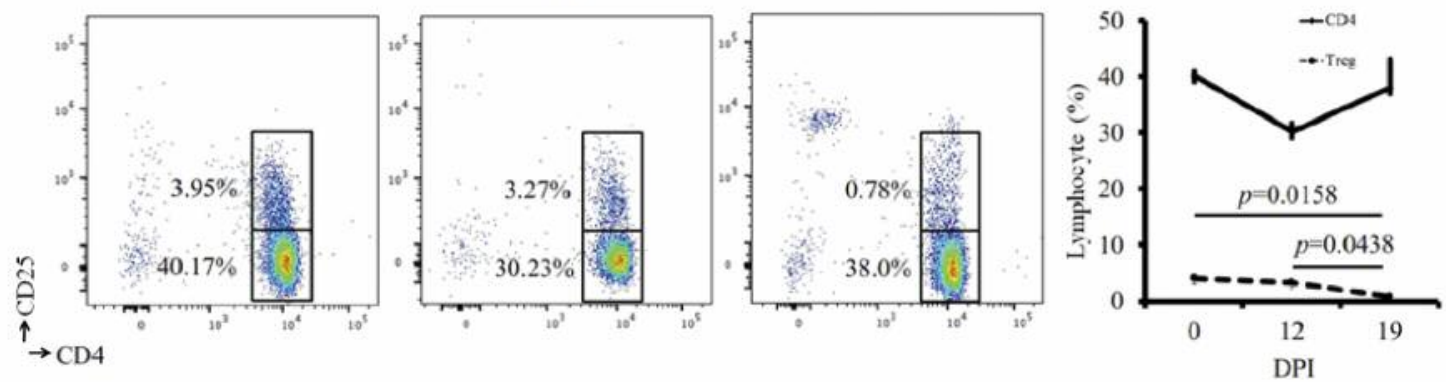

D

B-cell / dendritic cell

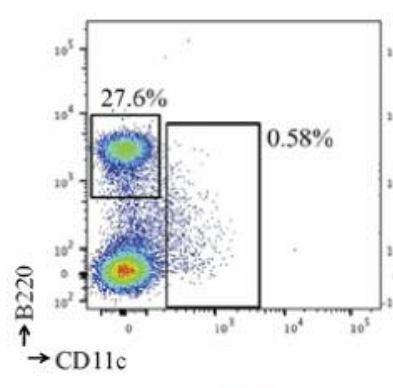

Day 0

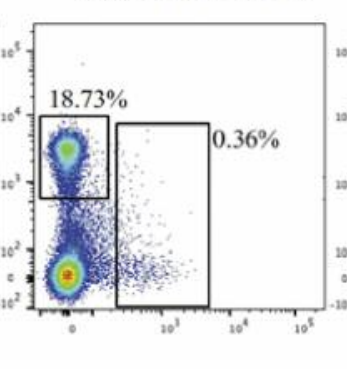

Day 12

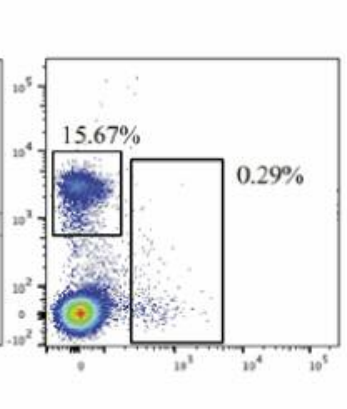

Day 19

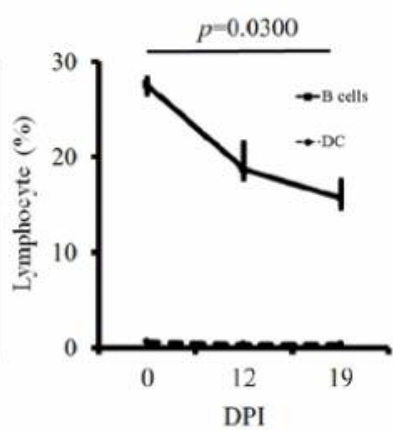

Figure 1. Myeloid-derived suppressor cells (MDSCs) substantially increased in the peripheral blood of tumor-bearing mice. Female C57BL/6 mice $(n=5)$ were subcutaneously (s.c.) inoculated with $1 \times 10^{7}$ B16F10 melanoma cells in the right flank. The following immune cell subsets in the peripheral blood were measured on the days indicated by flow cytometry: CD11b $\times$ granulocyte differentiation antigen $(G r 1)(A), C D 3 \times C D 8(B)$, $C D 4 \times C D 25(C)$, and CD11c $\times$ B220 (D). Left panels, Representative flow cytometric data; right panels, enumeration of the flow cytometric data. Each experiment was repeated 3 times; values are expressed as mean with 75 and 25 percentiles. $p$-Values were based on one-way analysis of variance with Holm's post-hoc test. A value of $p<0.05$ was considered statistically significant. DPI, Days post-tumor cell inoculation. 
plasma levels of GCSF, MCP1, and IL6 increased over time along with the tumor progression $(p=0.0234$ for G-CSF, $p=0.0618$ for MCP1, and $p=0.0120$ for IL6; Figure 3, upper panels). In contrast, IFN $\gamma, \mathrm{TNF} \alpha$, and IL17A exhibited no remarkable changes $(p=0.4185$ for IFN $\gamma, p=0.8253$ for $\mathrm{TNF} \alpha$, and $p=0.3818$ for IL17A; Figure 3, lower panels). These data suggest that MDSC-relevant cytokines such as GCSF, MCP1, and IL6 are preferentially induced in tumor-bearing mice.

Frequency of MDSCs in the peripheral blood reflected the status of tumor recurrence. The above findings (shown in Figures 1-3) clearly indicate that the frequency of MDSCs in the peripheral blood and the plasma levels of MDSC-relevant cytokines reflect the status of tumor progression. Based on these findings, we sought to address whether these factors would also reflect the status of tumor recurrence. To this end, we resected s.c. tumors on day 12 and monitored the mice for local tumor recurrence. The mice were then subgrouped into those with and those without local recurrence (Figure 4A). The frequency of MDSCs in the peripheral blood steadily increased until tumor resection on day 12. After resection, the frequency of MDSCs decreased. Nevertheless, mice with tumor recurrence exhibited an increase in the frequency of MDSCs, and they exhibited tumor recurrence when the frequency reached approximately $30 \%$ of total PBMCs. We reorganized the MDSC data based on the following phases: pre-recurrence, ortho-recurrence (at the time of recurrence), and post-recurrence ( $p=0.0163$ for pre $v s$. ortho, and $p=0.0121$ for ortho $v s$. post; Figure 4B). We recorded significant differences in the frequency of MDSCs in the peripheral blood among groups. Consistent with the MDSC data, the plasma levels of GCSF, MCP1, and IL6 significantly increased after local recurrence $(p=0.0320$ for GCSF, $p=0.0059$ for MCP1, and $p=0.0002$ for IL6; Figure 4C). However, there was no change observed in the level of INF $\gamma, \mathrm{TNF} \alpha$, and IL17A among the phases (data not shown). Taken together, these data suggest that the frequency of MDSCs and certain MDSCrelevant cytokines in the peripheral blood reflect the status of tumor recurrence. The tumor recurrence rate in these experiments was approximately $66 \%(n=24 / 36)$. We observed no significant differences in the volume of primary tumors prior to the resection among the groups (data not shown).

\section{Discussion}

The purpose of this study was to address whether the frequency of MDSCs in the peripheral blood reflects the pathological condition of tumor progression (particularly tumor recurrence) over time. We observed that the frequency of MDSCs in the peripheral blood was associated with macroscopic recurrence of s.c. tumors (Figure 4). Requisite to detecting tumor recurrence is that data correlate with the timing of tumor recurrence, that data are traceable over time,

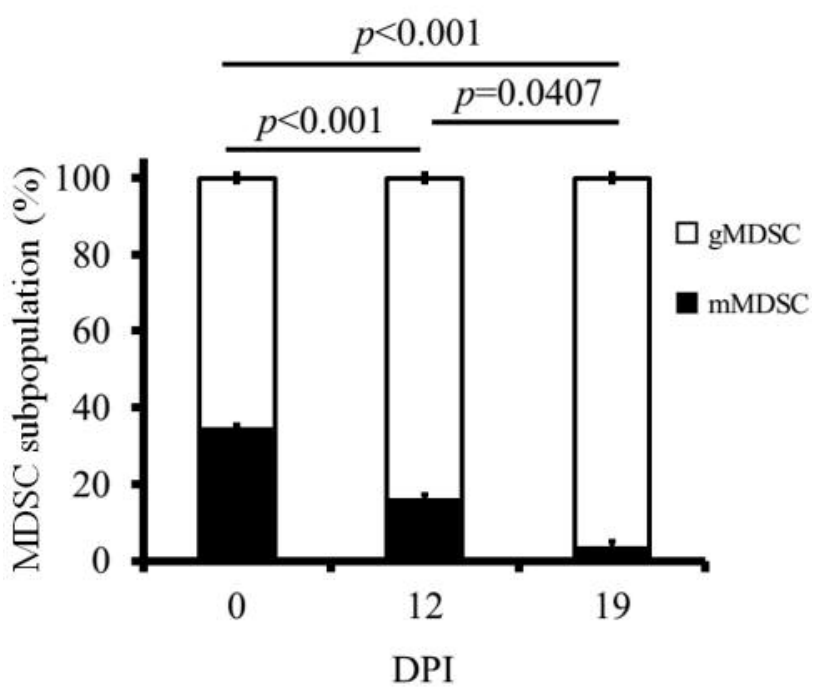

Figure 2. Granulocytic myeloid-derived suppressor cells (MDSCs) preferentially increase in tumor-bearing hosts. MDSC subsets were further evaluated on the days indicated. gMDSC: CD11 bi Gr 1mid Ly6Clo Ly6G hi $^{\text {hi }}$ granulocytic MDSCs. mMDSC: CD11 bigh Gr $1^{\text {mid }}$ Ly6Chi Ly6Glo monocytic MDSCs. p-Values were based on one-way analysis of variance with Holm's post-hoc test. A value of $p<0.05$ was considered statistically significant. DPI, Days post-tumor cell inoculation.

and that methods are feasible. From this viewpoint, the frequency of MDSCs in the peripheral blood is a useful indicator of tumor recurrence.

We previously reported that MDSCs accumulate in the lungs and spleen with the tumor progression in tumorbearing mice (9). Regarding the subsets of MDSCs, gMDSCs accumulated predominantly in these organs, whereas mMDSCs accumulated in the tumor microenvironment. Consistent with these observation, gMDSCs were found to increase systemically with tumor progression (Figures 1 and 2) as well as tumor recurrence (Figure 4A and B). These data suggest that the frequency of MDSCs in the peripheral blood is a useful indicator of tumor recurrence. It is clinically important to establish biomarkers to predict tumor recurrence because it allows individual tailor-made therapeutic strategies. Various biomarkers of tumor recurrence have been proposed thus far (7); most of them require expensive, time-consuming, special facility-requiring, cumbersome analyses. Compared to those previously proposed, it seems practically feasible to monitor the frequency of MDSCs in the peripheral blood.

In contrast, other cell populations exhibited no remarkable changes with the tumor progression (Figure 1B-D). Of note, Tregs have been shown to increase in the peripheral blood of tumor-bearing animals (2), which was not observed in this study. The kinetics of Tregs in the peripheral blood remains to be elucidated as an indicator of tumor recurrence. 

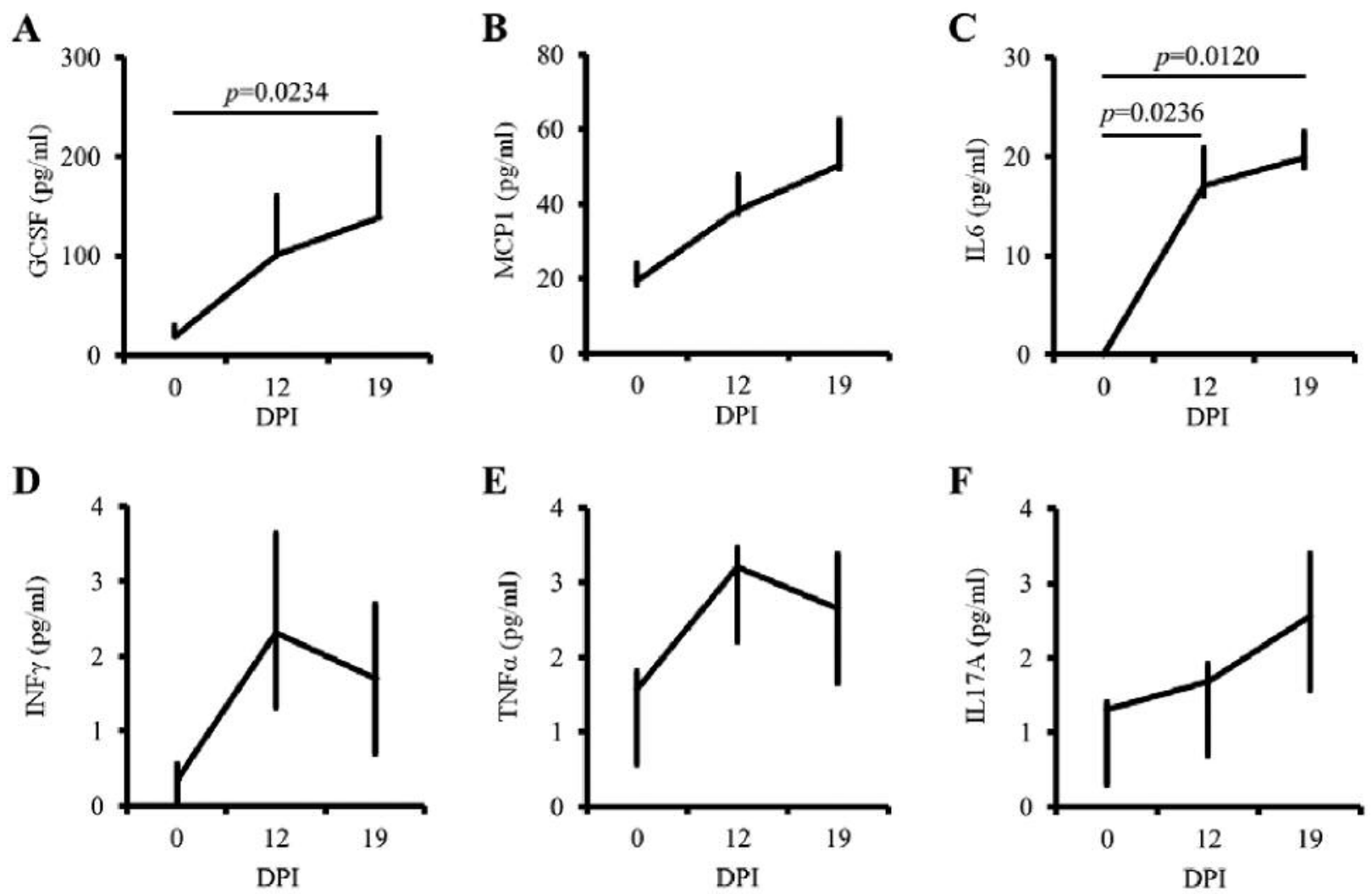

Figure 3. Myeloid-derived suppressor cells (MDSC)-relevant pro-inflammatory cytokines increase in tumor-bearing hosts. Plasma was collected from the peripheral blood of the tumor-bearing mice $(n=3)$. The following cytokines in the plasma were measured by cytokine bead array (CBA) and flow cytometry: granulocyte colony-stimulating factor (GCSF), monocyte chemoattractant protein 1 (MCP1), tumor necrosis factor alpha $(T N F \alpha)$, interleukin 6 (IL6), Interferon $\gamma(I F N \gamma)$, and interleukin 17A (IL17A). Each experiment was repeated 3 times; values are expressed as mean with 75 and 25 percentiles. $p$-Values were based on one-way analysis of variance with Holm's post-hoc test. A value of p $<0.05$ was considered statistically significant. DPI, Days post-tumor cell inoculation.

Pro-inflammatory cytokines are essential factors for the establishment of an immunosuppressive tumor microenvironment (13). Among them, GCSF is known to be produced by tumor-initiating cells and play important roles in the early stage of tumor progression (14). Consistent with these findings, the plasma levels of GCSF, MCP1 and IL6 (Figure 3A-C) clearly increased with tumor progression (Figure 3), as well as with tumor recurrence (Figure 4C) in this study. The problem in measuring these cytokines in order to predict tumor recurrence is that the cytokine changes are not as sensitive as MDSCs (Figure 4B) for this purpose. These results directed us to investigate the cell populations that produce GCSF, MCP1 and IL6, including MDSCs and Tregs.

We successfully established an animal model of tumor recurrence (Figure 4) using $1 \times 10^{7}$ B16F10 melanoma cells. In this regard, we previously observed no tumor recurrence when $1 \times 10^{5}$ cells were s.c. inoculated; when $5 \times 10^{6}$ cells were inoculated, about $20 \%$ of mice exhibited tumor recurrence (9). Given that we observed no metastasis to the lung throughout these experiments in this study, these observations collectively suggest that the size of primary tumors prior to resection is associated with local tumor recurrence. The animal model used in this study theoretically allows comprehensive analyses to identify predictive factors for tumor recurrence and to determine therapeutic effects. We are currently improving the analysis system to this end.

In summary, we demonstrated that the frequency of MDSCs in the peripheral blood of hosts after tumor resection is an appropriate indicator of tumor recurrence. Clinically, early detection of tumor recurrence allows individual tailormade therapeutic strategies and might lead to complete cure from malignant tumor. From this viewpoint, our data suggest that continuous monitoring of MDSCs seems to be a feasible strategy for detecting tumor recurrence at the early phase. Further studies will be necessary to determine whether the continuous monitoring of MDSCs in humans would be useful in detecting tumor recurrence after curative resection. 
A

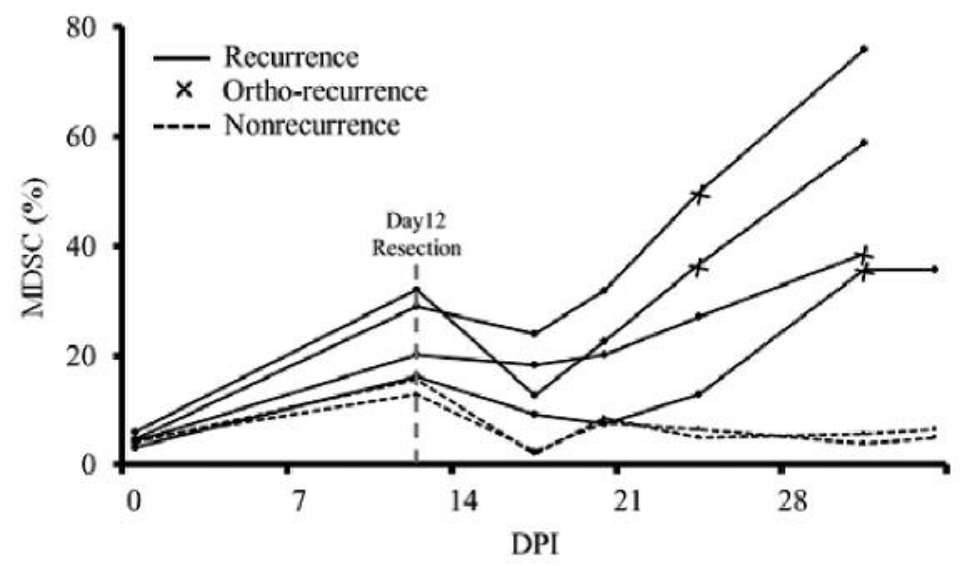

C

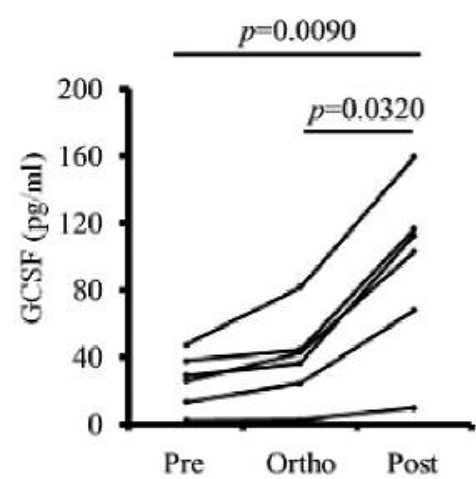

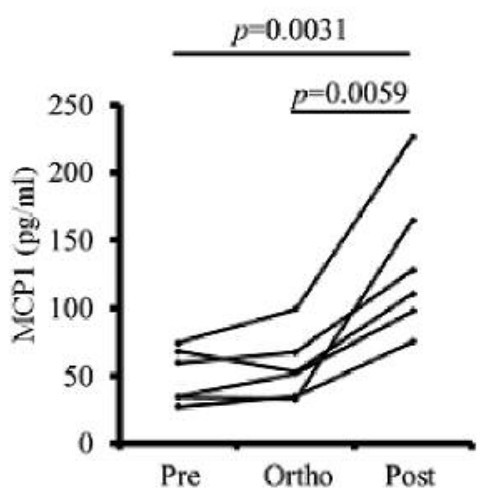

B
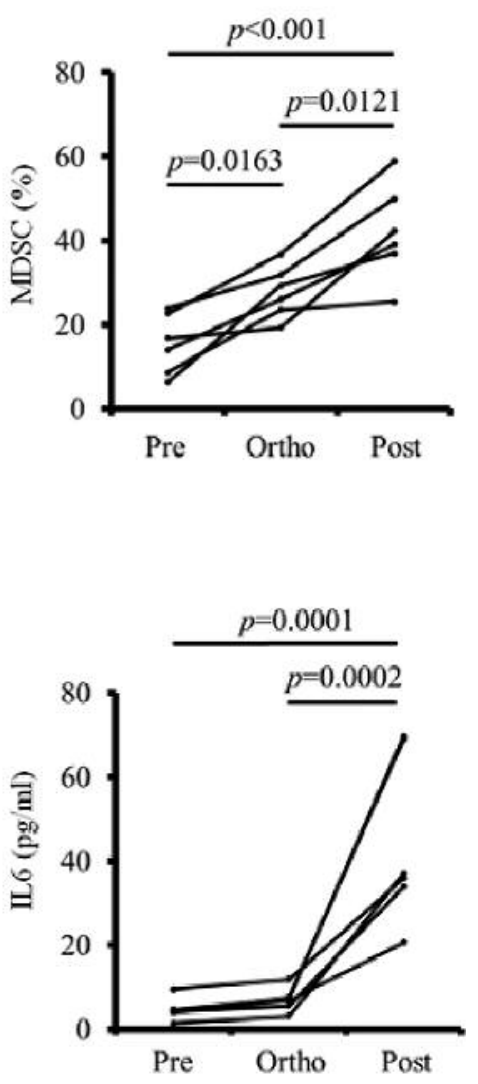

Figure 4. Frequency of myeloid-derived suppressor cells (MDSCs) in the peripheral blood reflects the status of tumor recurrence. A: The s.c. tumors were surgically removed on day 12 after tumor cell inoculation. The mice were periodically monitored for local recurrence and subgrouped based on tumor recurrence; the frequency of MDSCs in peripheral blood was measured by flow cytometry and plotted. B: The frequency of MDSCs in the peripheral blood of the mice with tumor recurrence was plotted based on the following phases: pre-recurrence, ortho-recurrence, and postrecurrence. C: Plasma levels of interleukin 6 (IL6), granulocyte colony-stimulating factor (GCSF), and monocyte chemoattractant protein 1 (MCP1) were simultaneously measured. -Values were based on one-way ANOVA with Holm's post-hoc test. A value of $p<0.05$ was considered statistically significant. DPI, Days post-tumor cell inoculation.

\section{Acknowledgements}

All the Authors have no conflict of interest in regard to this study. This work was supported by a Grant-in-Aid from the Ministry of Education, Culture, Sports, Science, and Technology of Japan (KY: No. 16K10541; YK: No. 16H05415).

\section{References}

1 Rabinovich GA, Gabrilovich D and Sotomayor EM: Immunosuppressive strategies that are mediated by tumor cells. Annu Rev Immunol 25: 267-296, 2007.

2 Tanaka A and Sakaguchi S: Regulatory T-cells in cancer immunotherapy. Cell Res 27: 109-118, 2017.

3 Gabrilovich DI and Nagaraj S: Myeloid-derived suppressor cells as regulators of the immune system. Nat Rev Immunol 9: 162$174,2009$.
4 Kumar V, Patel S, Tcyganov E and Gabrilovich DI: The nature of myeloid-derived suppressor cells in the tumor microenvironment. Trends Immunol 37: 208-220, 2016.

5 Sharma P and Allison JP: The future of immune checkpoint therapy. Science 348: 56-61, 2015.

6 Restifo NP, Smyth MJ and Snyder A: Acquired resistance to immunotherapy and future challenges. Nat Rev Cancer 16: 121126, 2016.

7 Chi KR: The tumour trail left in blood. Nature 532: 269-271, 2016.

8 Yamashita K, Hasegawa H, Fujita M, Nishi M, Tanaka T, Arimoto A, Suzuki S, Kamigaki T and Kakeji Y: Host CD40 is essential for DCG treatment against metastatic lung cancer. Anticancer Res 36: 3659-3665, 2016.

9 Otsubo D, Yamashita K, Fujita M, Nishi M, Kimura Y, Hasegawa H, Suzuki S and Kakeji Y: Early-phase treatment by low-dose 5-fluorouracil or primary tumor resection inhibits MDSC-mediated lung metastasis formation. Anticancer Res 35: 4425-4431, 2015. 
10 Hasegawa H, Yamashita K, Otubo D, Fujii S, Kamigaki T, Kuroda D and Kakeji Y: Allogeneic DCG promote lung NK cell activation and antitumor effect after invariant NKT cell activation. Anticancer Res 34: 3411-3417, 2014.

11 Tasaki T, Fujita M, Okuda T, Yoneshige A, Nakata S, Yamashita $\mathrm{K}$, Yoshioka $\mathrm{H}$, Izumoto $\mathrm{S}$ and Kato A: MET expressed in glioma stem cells is a potent therapeutic target for glioblastoma multiforme. Anticancer Res 36: 3571-3577, 2016.

12 Hasegawa H, Yamashita K, Otsubo D and Kakeji Y: Liver injury after invariant NKT cell activation by free alphagalactosylceramide and alpha-galactosylceramide-loaded dendritic cells. Anticancer Res 36: 3667-3672, 2016.

13 Dranoff G: Cytokines in cancer pathogenesis and cancer therapy. Nat Rev Cancer 4: 11-22, 2004.
14 Welte T, Kim IS, Tian L, Gao X, Wang H, Li J, Holdman XB, Herschkowitz JI, Pond A, Xie G, Kurley S, Nguyen T, Liao L, Dobrolecki LE, Pang L, Mo Q, Edwards DP, Huang S, Xin L, Xu J, Li Y, Lewis MT, Wang T, Westbrook TF, Rosen JM and Zhang XH: Oncogenic mTOR signalling recruits myeloidderived suppressor cells to promote tumour initiation. Nat Cell Biol 18: 632-644, 2016.
Received March 5, 2017

Revised April 5, 2017

Accepted May 5, 2017 